\title{
Effectiveness of Pedagogical Tools for Teaching Medical Gross Anatomy During the COVID-19 Pandemic
}

\author{
Dustin C. Lin ${ }^{1}$ (D) Bailey Bunch ${ }^{1} \cdot$ Raissa Zuim Dantas De Souza ${ }^{1} \cdot$ Dickson Chen $^{1} \cdot$ Jinyan Zhou ${ }^{1} \cdot$ Ann C. Zumwalt ${ }^{1}$. \\ Jonathan J. Wisco'
}

Accepted: 9 February 2022 / Published online: 24 February 2022

(c) The Author(s) under exclusive licence to International Association of Medical Science Educators 2022

\begin{abstract}
Medical institutions have been forced to modify gross anatomy pedagogy to comply with the health restrictions imposed by the novel coronavirus (COVID-19). Boston University School of Medicine (BUSM) is one such institution that temporarily restructured its course. We replaced cadaveric dissection activities with prosections and placed a greater emphasis on a flipped classroom model. This study investigates the effectiveness of new course materials developed to aid these curriculum changes. Course materials were developed for three purposes: (1) preparation before laboratory sessions (orientation videos and Complete Anatomy (3D4Medical, Elsevier) screens); (2) guidance during laboratory sessions (laboratory guides); and (3) review after laboratory sessions (Zoom recitation sessions). We performed a grounded theory thematic analysis of students' responses (80/160, 50\% response) to qualitative survey questions and to focus group questions (16 students who self-selected between 4 different sessions). Data from both the survey and focus groups demonstrated that the vast majority of students agreed that the materials helped them navigate through learning gross anatomy. However, laboratory guides were used mostly for post-lab review as opposed to the intended direction during laboratory sessions. Students within all focus groups overwhelmingly touted the value of Zoom recitation sessions, with many stating that they were imperative to course success. When comparing performance data between 2019 (pre-COVID) and 2020 students, we found that the students who took the anatomy course during the onset of COVID had a slightly higher overall average score in all three modules of the course than compared to the 2019 students. We propose that the utilization of course materials that students perceived as time saving and pertinent to their exam performance, when combined with cadaveric prosection, emphasized the benefits of flipped-classroom learning to help students learn gross anatomy effectively and efficiently during the pandemic and beyond.
\end{abstract}

Keywords Medical education · Gross anatomy education · COVID-19 pandemic · Flipped classroom · Online teaching

\section{Introduction}

Gross anatomy is one of the first classes medical students usually take during their career but it is also a foundational aspect of future clinical practice. A concrete understanding of anatomy has been acknowledged to be important for safe clinical practice and fundamental for teaching problem solving $[1,21,31]$. The traditional medical anatomy course is divided into two major components, consisting of a didactic

Dustin C. Lin and Bailey Bunch contributed equally to this work.

Dustin C. Lin

dclin@bu.edu

1 Department of Anatomy and Neurobiology, Boston University School of Medicine, Boston, MA 02118, USA lecture and a dissection-focused laboratory session. Cadavers have been used as the main instructional tools in gross anatomy for hundreds of years and as such most laboratory components center around their usage [8].

The classic subject of debate is whether learning gross anatomy is best accomplished through prosection or dissectionbased courses. Historically, cadaveric dissection has played the major role in disseminating anatomy curriculum to medical students. Many studies strongly advocate dissection as the preferred learning method [2, 3, 25]. Engaging in dissection allows the student to understand relational anatomy that may be difficult to conceptualize through prosections and virtual learning platforms. However, some argue that student learning of anatomy is not dependent on dissection [30]. Prosection has also been shown to be a very effective method of learning gross anatomy [19]. Some note 
that prosection is sufficient but dissection experience is necessary in preparation for the anatomical medical specialties [34]. Others claim that cadavers can be dispensed altogether and replaced by other contexts, such as an earlier introduction to medical imaging [18].

As long as programs choose to use cadavers, regardless of whether they utilize a dissection or prosection based laboratory component, the novel coronavirus (COVID-19) has elicited real impacts in many departments about the safety of continued body donation programs. Donors who screened negative for COVID-19 prior to death is not sufficient to rule out infection [33]. However, guidance from the CDC suggests that the most common modes of transmission of the SARS-CoV-2 virus are through direct, indirect, or close contact with bodily secretions, respiratory droplets, and fomites expelled from an infected person [23]. In the context of anatomical donors, guidelines from the International Committee of the Red Cross recommends transmission-based precautions such as personal protective equipment (PPE) in the handling of donor bodies that may or may not have been affected by COVID-19 [9]. Similar PPE guidelines pertaining to the procedures of handling donor bodies have historically been mandated by medical institutions and followed by medical students across the world. Cytopathological studies suggest that common preservatives for body donors such as formaldehyde and ethanol may be effective against COVID19 , but there is no concrete evidence if the virus would be inactivated [17, 22, 28].

As such, proper precautions needed to be taken to ensure the safety of the community while also upholding an appropriate medical education for our future health care professionals. In recent years, gross anatomy curricula have already seen a decrease in the hours devoted to teaching anatomy $[6,7,32]$. COVID safety protocols have reduced the number of individuals allowed in the laboratory space as well as the continued donation of cadavers [10, 26], which created significant restrictions on the amount of time and options available for the cadaveric anatomy learning experience. The lack of opportunities to dissect and to meet with the families of the donors also makes it more difficult to reinforce respectful and professional attitudes $[5,11]$. The motivation to redesign the laboratory experience was driven as much, if not more, by the need to control how students interact with one another safely in the laboratory space. Students were divided into cohorts to allow for contact tracing. They were prohibited from mingling in the laboratory space as they typically would during dissection, nor were they permitted to enter the laboratory space during other times of the day. These restrictions forced the teaching staff at BUSM to modify how we teach since the community needed to abide by social distancing guidelines. The dissection-based laboratory course at the Boston University School of Medicine (BUSM) was redesigned in order to accommodate the challenges presented by COVID-19. Primary changes included a greater emphasis on a flipped classroom model and the utilization of prosected cadavers to replace cadaveric dissection activities. Prosected cadavers are specimens that experienced anatomists dissect and prepare for students to study. Numerous novel anatomy pedagogical tools were also designed in order to accommodate the restructured course at BUSM. These new pedagogical tools included orientation videos, Complete Anatomy screens, laboratory guides, and Zoom recitation sessions. This article observes the degree to which students perceive the effectiveness of learning tools in reference to medical gross anatomy. Connectivism learning theory describes learning as influenced by technology and socialization [12]. COVID-19 has increased the utilization of educational technology and the amount of flipped classroom learning across the world. We hypothesize connectivism learning theory now plays a more substantial role than before in disseminating gross anatomy curriculum during COVID19. In anticipation of the changes necessary to maintain student safety in the lab, design of the new curricular resources and pedagogical techniques were borne from forethought of learning theory. We produced and implemented the use of these resources with new pedagogical techniques specific to connectivism theory that students need technological tools to engage in social learning. Behaviorism and cognitivism theory would not have necessitated the creation of new tools as we have described to provide a robust learning experience for the students. We examined student perceptions of the effectiveness of these newly created course materials at BUSM. We propose that the benefits of flipped-classroom learning are augmented via the utilization of these course materials and this allows BUSM students to maintain learning of gross anatomy content.

\section{Course Description}

The medical anatomy class at the Boston University School of Medicine (BUSM) is divided into three modules. The three modules are named Body Structures 1 (BS1) or Back \& Limbs; Body Structures 2 (BS2) or Thorax, Abdomen, \& Pelvis; and Body Structures 3 (BS3) or Head \& Neck. The course is 9 weeks long, with each body structure module taking up 3 weeks of time. To comply with COVID-19 safety protocols, students were divided into three cohorts, termed cohorts 1, 2, and 3 respectively. All cohorts attended lecture via Zoom. Students were primarily assessed through lecture examinations and laboratory practicals, both of which were administered at the end of each module online through the Blackboard learning management system. BS1 and BS2 modules were organized in a similar fashion whereas BS3 was arranged differently. 
In BS1 and BS2, lectures were held every week leading up to the lecture examination. Students from each respective cohort attended laboratory sessions once a week. Sessions were $2 \mathrm{~h}$ in duration. Students were expected to preview for laboratory sessions by watching orientation videos and studying the provided Complete Anatomy screens. During laboratory sessions, students were provided with laboratory guides. These guides included a list of relevant structures that students are expected to identify. The objective of weekly laboratory sessions was to identify structures using assistance from laboratory guides, prosectors, and faculty, and by engaging in meaningful discussion. On the third week, students were assessed and graded in laboratory sessions through table conferences. Table conferences are oral examinations administered by prosectors.

In BS3, all lectures were provided to students on the first week. Students do not attend a laboratory session during the first week of BS3. Students attended laboratory sessions on the second and third week of the BS3 module. The session during the second week was $4 \mathrm{~h}$ in duration and the session during the third week was $2 \mathrm{~h}$ in duration. The expectations during BS3 are the same as BS1 and BS2. Students are likewise assessed through table conferences. To review laboratory material, students were expected to use lecture material, laboratory guides, orientation videos, Complete Anatomy screens, and other provided resources. Additionally, students were given the option to attend Zoom recitation sessions which are review sessions held by prosectors. In comparison, students pre-COVID had $4 \mathrm{~h}$ of structured lab per week as well as 24/7 access to the lab.

\section{Laboratory Layout}

The laboratory room itself is divided into four pods (Fig. 1). Each pod contains six tables or stations. The material present at each table varies depending on the module (i.e., BS1,
BS3, or BS3). Tables one to four contain cadaveric prosections. Table five is designated as the osteology table and where osteology guides are utilized. Table six varied as a rest station where students discuss clinically related material. As previously stated, students in respective cohorts attend these $2 \mathrm{~h}$ ( $20 \mathrm{~min}$ at each of the six stations) laboratory sessions once per week. Faculty and prosectors are stationed at each pod and present to answer student questions. Laboratory guides present in the form of PDFs on iPads are utilized at the stations of each pod.

\section{Orientation Videos}

It was necessary to provide students with prior exposure to laboratory materials due to the markedly reduced amount of time available for them to be in the cadaver laboratory due to COVID safety restrictions. Orientation videos were short pre-laboratory videos designed with the intent to "orient" or introduce the student to the relevant structures they would be viewing in the cadaver laboratory. Not all structures that needed to be viewed in laboratory sessions were explained in the orientation videos. Orientation videos showcased anatomical structures using a combination of cadaver images and the 3D human anatomy software, Complete Anatomy. In these videos, a narrator explained the relationship between structures and gave pointers to help students with identification while the images are displayed. The videos range from 3 to $7 \mathrm{~min}$ in duration and were created for all modules of the class. For the BS1 module (back and limbs), the following orientation videos were created: (1) Upper Extremity; (2) Lower Extremity. For the BS2 module (Thorax, Abdomen, \& Pelvis), the following orientation videos were created: (1) Trunk Wall; (2) Abdominal Organs; (3) Abdominal Vasculature; (4) Perineum, Pelvic Organs, and Neurovasculature. For the
Fig. 1 Laboratory pods layout:

This shows the organization of the four pods in the gross anatomy lab room for Body Structures 1. Students rotate tables after spending the allotted time identifying structures or completing assigned activities

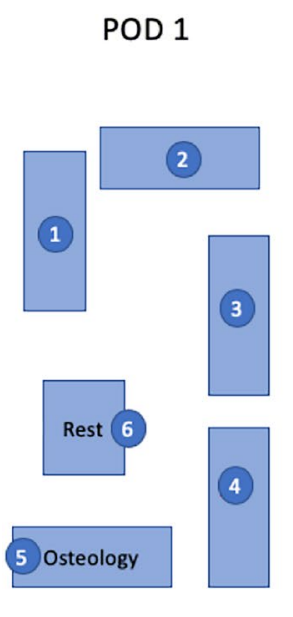

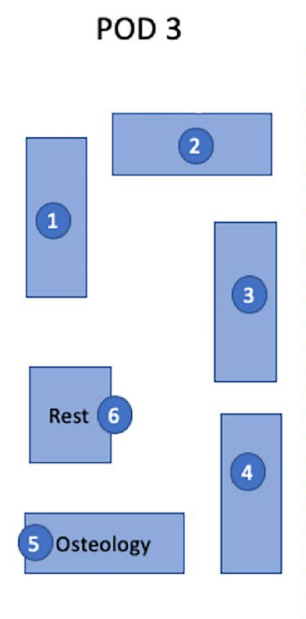

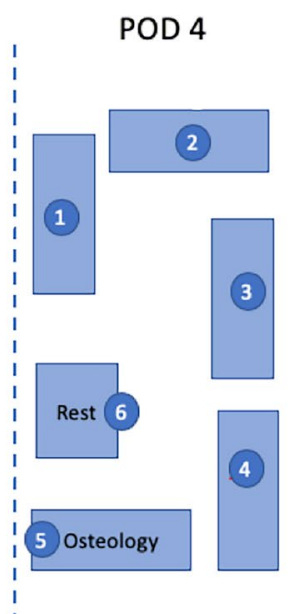


BS3 module (Head and Neck), the following orientation videos were created: (1) Neck; (2) Cranial Nerves in the Neck; (3) Retropharyngeal Space; (4) Infratemporal Fossa.

\section{Complete Anatomy Screens}

Complete Anatomy is an educational anatomy program used by students to explore 3D anatomy models. The application has been shown to be useful for supporting laboratory session curriculum transition to an online learning modality (Havens 2020). We created unique screens for the topics covered in the laboratory in every week of all three body structure modules. The resultant screens were interactive in that a learner can rotate the 3D models to explore different orientations using a mouse on the computer or fingers on a mobile device. Clicking the structure displays additional information pertaining to that structure.

\section{Laboratory Guides}

Whereas the orientation videos were designed to be used prior to laboratory sessions, the laboratory guides were instructional manuals designed with the intent to be utilized during the lab in order to guide students towards identifying structures. The guides contain both cadaver images and non-cadaver images with succinct descriptions of each structure. Images were used from Rohen et al. [27]. Different laboratory guides were created that corresponded to each prosection station during laboratory sessions. Each laboratory guide contained approximately 60 to 100 structures for a specific topic or region of the body (e.g., brachial plexus, abdominal vasculature, orbit, retropharyngeal space). Students were expected to read and study the laboratory guides during their allotted time at the station. Laboratory guides were available in PDF format on iPads that were given to each group of students. For the BS1 module (back and limbs), the primary laboratory guides created were Back and Shoulder, Arm and Axilla, Neurovasculature of Upper Extremity, Knee, Anterior Leg, Lateral Leg, Gluteal Region, Posterior Thigh, and Laminectomy. For the BS2 module (Thorax, Abdomen, \& Pelvis), the primary laboratory guides created were Heart, Lung, Thoracic Spaces, Thoracic Cavity with Heart in Situ, Body Wall, Peritoneum, Abdominal Vasculature, Male Pelvic Organs, and Female Pelvic Organs. For the BS3 module (Head and Neck), the following laboratory guides were created: Brain, Cranial Vault, Cranial Nerves, Eye, Orbit, Infratemporal Fossa, and Deep Neck.

\section{Zoom Recitation Sessions}

Recitation sessions were online synchronous sessions provided by lab prosectors who are fourth year medical students and graduate students. Attendance was not mandatory. Sessions ranged anywhere from five students to twenty students per recitation. Review sessions in the medical anatomy course are traditionally held in the laboratory which allows prosectors to instruct on the cadavers and anatomy models. Due to social distancing restrictions, in Fall 2020, all review sessions were transitioned to remote learning on the video conferencing software Zoom. These sessions were designed with the intent to review laboratory material and help students identify structures on cadaveric images. The format of the session and the content covered was dependent on the instructor. However, students had the ability to suggest topics to be covered and the style of the review session (e.g., rapid review, multiple choice, clinically orientated). Recitation sessions also covered content from lecture and were available in a question and answer format. A large number of sessions were held throughout the semester. Reviews were held every day of the week ranging from early morning to late evening.

\section{Methods}

We conducted both an online survey and focus groups at Boston University School of Medicine (BUSM) for BUSM medical students and Anatomy and Neurobiology (A\&N) students in 2020 to assess students' attitudes towards the effectiveness of these new anatomical pedagogical tools. The Anatomy and Neurobiology participants are 1st year graduate students. They are included because they take the same gross anatomy course and receive the same assessments as the medical students. The survey was distributed online through Qualtrics and focus groups were administered via Zoom.

This initial recruitment email provided instructions to the participants regarding their identity:

Your participation or lack thereof will not influence your grades or eligibility for any programs. If you are selected to be in a focus group, we will ask everyone in the focus group not to talk about the discussions outside the group. However, we can't promise that everyone will keep what you say confidential. Audio recordings of the focus groups sessions will be made. We will not record your name or any information that shows your identity. You will not be signing this form. We will store electronic files in computer systems with password protection and encryption. However, we cannot guarantee complete confidentiality. 
The survey was divided into three sections. It consisted of general questions, resource-specific questions, and open-ended responses. The general questions collected demographic information about the student and asked them which resources they used during class. Conditional branching within the survey leads to the resource-specific questions based on the participants' answer choice. The resourcespecific questions assess several aspects of the particular virtual resources the student used. Answers to the resource-specific questions were classified as strongly disagree, disagree, neutral, agree, and strongly agree (range 1-5 on Likert scale). Responses were considered to be positive if the answer chosen was agree or strongly agree ( $>3.6$ rating). The final section of the survey was an optional open-ended response which allowed the participant to relay any additional comments. The survey took approximately $10 \mathrm{~min}$ to complete.

Students' responses to quantitative survey questions were analyzed using Spearman's rank-order correlation in SPSS (version 27; IBM, Inc.). Likert scale data was analyzed using Spearman's correlation based on three assumptions: (1) chosen variables were measured on an ordinal scale and therefore non-parametric; (2) variables represented paired observations; and (3) variables displayed a monotonic relationship. We chose a 2-tailed alpha level of 0.05. Using Spearman's test, we explored the correlation between the following variables: (1) orientation videos and laboratory guides; (2) orientation videos and Complete Anatomy screens; and (3) Complete Anatomy screens and laboratory guides. A high value of Spearman's rho indicates a strong positive correlation between variables.

From among the respondents of the survey, 16 participants volunteered to participate in four focus groups over Zoom. There were four, five, six, and one student respectively in each focus group. The focus groups were administered immediately following the last examination of the course. Within the focus groups, we asked students questions regarding the course materials described in this study and for the previously mentioned questions, we quoted student answers. We performed a grounded theory thematic analysis of students' responses to both qualitative survey questions and to focus group questions [20]. Six raters were used for the thematic analysis. In this process, de-identified audio recordings were heuristically coded for frequent and emphasized phrases, which were subsequently categorized into themes. After the themes were identified by individual raters, our team of raters discussed the significance of each theme, and returned to the coded data for supporting quotes.
In this article, we analyzed questions relevant to the course materials that pertain to questions $1,2,3$, and 13 .

$* 1$. What aspects of the orientation videos were most helpful and what was less helpful?

*2. What aspects of the Complete Anatomy screens were most helpful and what was less helpful?

*3. What aspects of the lab guides were most helpful and what was less helpful?

*13. What aspects of the Zoom recitation sessions were most helpful and what was less helpful?

Additionally, in order to further gauge if students adequately learned the material, we compared data from both written and laboratory practical examinations from course results in 2019 and 2020. For this retrospective cohort comparison, we were guided by the BUSM IRB to access only class grades for each module in body structures for the 2 years; hence, we did not have access to individual grades for statistical analysis.

\section{Survey Results}

\section{Survey Demographics}

The 2020 medical gross anatomy course had a class size of 160 students with the vast majority ( $>95 \%$ ) comprised of first year medical students and the remaining comprised of Anatomy and Neurobiology (A\&N) graduate students (M.S. and Ph.D.). In all, 80 of the 160 (50\%) of the students completed the survey. The response rate from BUSM medical students was $83.95 \%(n=68)$. The response rate for Masters A\&N students was $12.35 \%(n=10)$. The response rate for PhD A\&N students was $2.47 \%(n=2)$. We recognize the somewhat low survey response rate as a limitation of this study. This may have been because the survey was emailed out during the time when the medical gross anatomy course had ended and the students were transitioning to their next course.

\section{Overview of Survey Responses}

Overall, most participants who responded to our survey considered the anatomical pedagogical resources created to be a valuable complement to learning gross anatomy and succeeding in the course. We determined that a positive response is considered any rating between 3.6 and 5.0 on a Likert scale.

Overall, students strongly agreed or agreed that the orientation videos, Complete Anatomy screens, and lab guides were effective aids in learning gross anatomy. Likert scale ratings were $3.82,4.02$, and 3.81 respectively for when the students were asked to rate their level of agreement to the following three statements (Fig. 2). Additionally, we looked 
Fig. 2 Horizontal bar graph for pedagogical resources. Number of students who strongly agreed or agreed with effectiveness of a orientation videos, $\mathbf{b}$ Complete Anatomy screens, and $\mathbf{c}$ laboratory guides
Orientation videos made it easier to identify cadaveric structures.

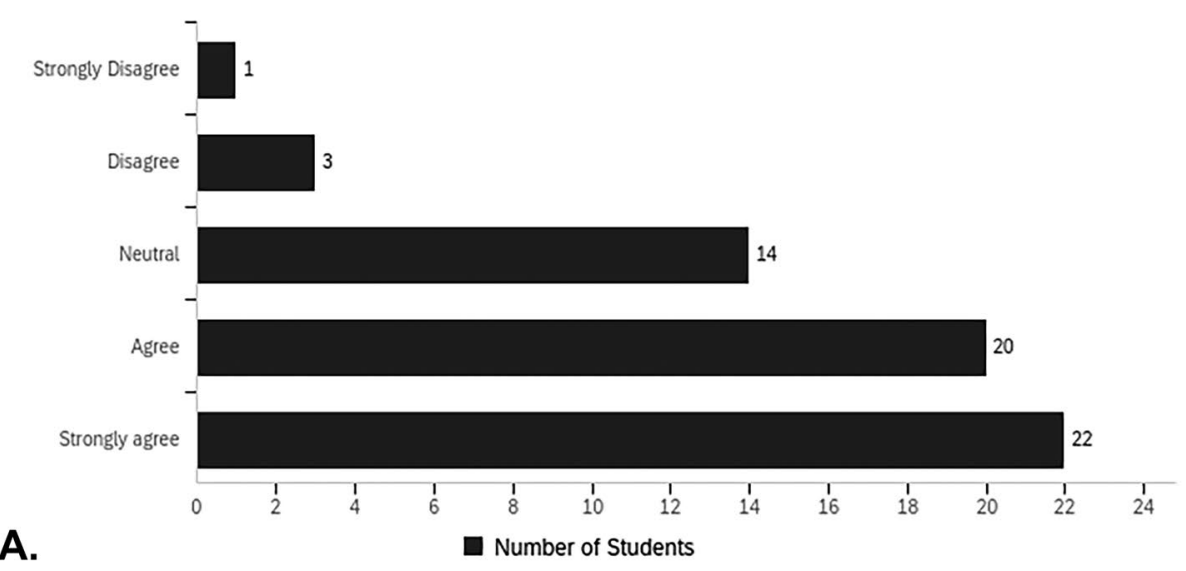

Complete Anatomy screens are an effective resource in learning gross anatomy.

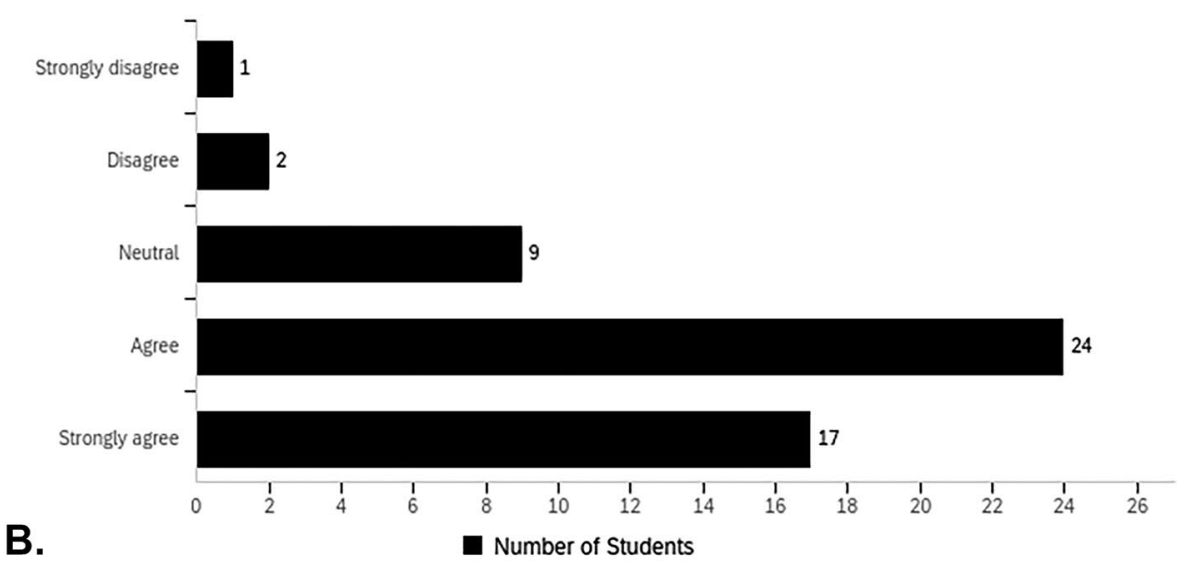

The lab guides prepared me for the lab practical.

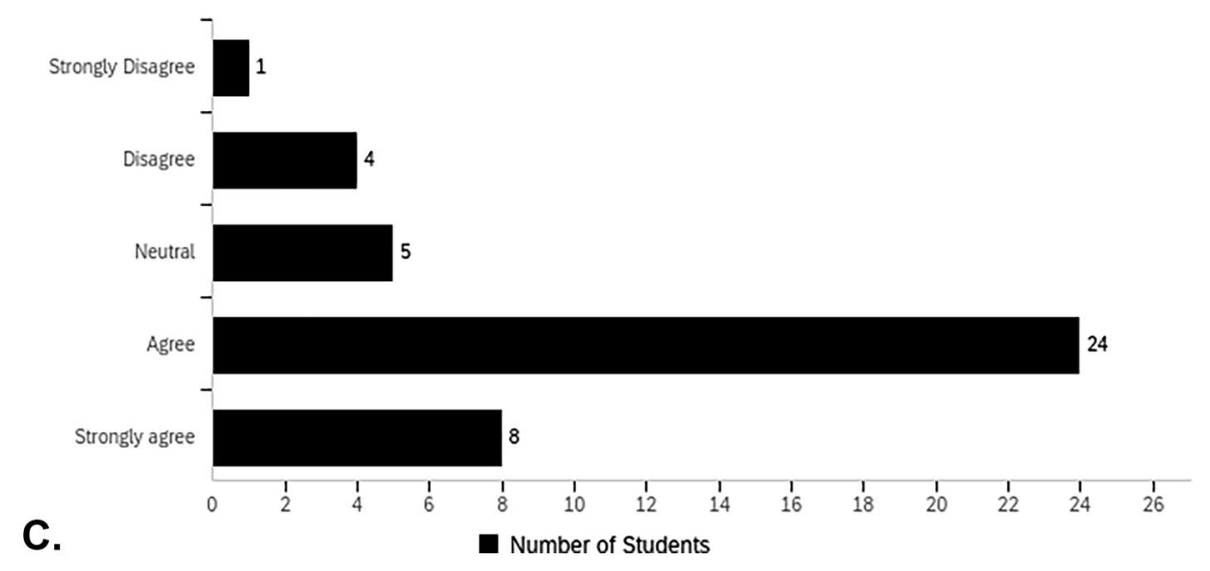


Table 1 Correlation between resources: Spearman's rho, significance (2-tailed), and 95\% confidence intervals (2-tailed) of all resources compared against one another

\begin{tabular}{lllll}
\hline & $\begin{array}{l}\text { Spearman's } \\
\text { rho }\end{array}$ & $\begin{array}{l}\text { Significance } \\
\text { (2-tailed) }\end{array}$ & \multicolumn{2}{l}{$\begin{array}{l}\text { 95\% confidence } \\
\text { intervals (2-tailed) }\end{array}$} \\
\cline { 4 - 5 } & & & Lower & Upper \\
\hline Orientation video vs Complete Anatomy screens & 0.734 & 0.000 & 0.549 & 0.850 \\
Orientation video vs lab guides & 0.344 & 0.043 & 0.002 & 0.614 \\
Lab guides vs Complete Anatomy screens & 0.430 & 0.016 & 0.078 & 0.686 \\
\hline
\end{tabular}

at correlations between ratings of all three resources in order to determine if students who rated one resource highly also rated other resources highly and found there to be a positive association between resources (Table 1).

\section{Correlation Between Effectiveness of Gross Anatomy Pedagogical Resources}

To determine the efficacy of implementing these gross anatomy pedagogical resources, a series of Spearman's rank-order correlations were performed to determine the best combination of resources that were considered most effective by the students. No significant positive correlation was found between orientation videos and lab guides $(\rho=0.734,0.549<\mathrm{CI}<0.850$; Fig. 3A). There was also no significant positive correlation found when evaluating the effectiveness of Complete Anatomy screens and orientation videos $(\rho=0.344, \mathrm{p}<0.05,0.002<\mathrm{CI}<0.614$; Fig. 3B $)$. In determining the effectiveness of Complete Anatomy screens and lab guides, no significant positive correlation was found $(\rho=0.430,0.078<\mathrm{CI}<0.686$; Fig. 3C).

\section{Focus Group Results}

1) What aspects of the orientation videos were most helpful and what was less helpful?

While participants across all four focus groups utilized the orientation videos in different capacities, overall students agreed that the orientation videos complemented their learning of gross anatomy both within the laboratory setting and in preparation for the laboratory practical. Most viewed the videos before laboratory sessions as intended. However, other students viewed the videos after sessions, both before and after sessions, or did not utilize the videos in any capacity. Students attributed the timing of their usage of video due to circumstance. One student attributed their usage of videos before or after sessions based on how caught up they were in class, while others who viewed the videos after sessions noted that the videos were "less helpful orientation and more helpful as review." This is due to the fact that depending on which laboratory session the student was registered for, they may or may not have had the corresponding lecture yet.

There were two aspects of the orientation videos that students found to be helpful. The first aspect is the video's intended purpose, that is to say, the "orientation" or introduction. Students agreed that the videos were beneficial in the regard that it introduced them to the relevant material that week. Some relevant quotes from students included: "Good way to see everything big picture, before you start going into the details," and "... having someone say it out loud so you could start that repetition process in your head was very helpful."

The second aspect was related to organization pertaining to the placement of cadaveric images alongside Complete Anatomy screens within the videos. Students strongly advocated for this organization. This arrangement allowed the student to make comparisons between cadaveric images and illustrations and gain a better awareness of what they are expected to observe during the lab. One student noted: “...illustrations and handdrawn images are useful; however, it is more difficult to extrapolate that information to real human body."

Number of orientation video views fell steadily as the course progressed. The average views per video in each module dropped from 182 views in Body Structures 1 (Back \& Limbs), 109 views in Body Structures 2 (Thorax, Abdomen, \& Pelvis), and 66 views in Body Structures 3 (Head \& Neck). We posed the following follow-up question to participants: "Did the quality of the videos decrease as the course progressed?" The responses indicated that this was not the case. Students agreed that adjusting to the language (vocabulary and diction) of anatomy is an initial challenge during the course. As the course progressed, students adapted learning styles that they believed were appropriate to succeed in the course. Numerous students noted that they "developed their own learning strategy as the course progressed."

Critiques of the videos were related to video duration. Currently the videos range from 3 to $7 \mathrm{~min}$ in duration. Some students expressed that they would have preferred shorter videos. They were apprehensive about having to watch longer videos due to the plethora of lectures 
Fig. 3 A: Scatterplot of orientation videos against lab guides: Spearman's rank-order correlation was used to analyze data between the effectiveness of orientation videos and lab guides. This scatterplot shows a positive correlation of the monotonic relationship between two non-parametric variables. B Scatterplot of orientation videos against screens: Spearman's rank-order correlation was used to analyze data between the effectiveness of orientation videos and screens. This scatterplot shows a positive correlation of the monotonic relationship between two non-parametric variables. C Scatterplot of lab guides against screens: Spearman's rank-order correlation was used to analyze data between the effectiveness of lab guides videos and Complete Anatomy screens. This scatterplot shows a positive correlation of the monotonic relationship between two non-parametric variables

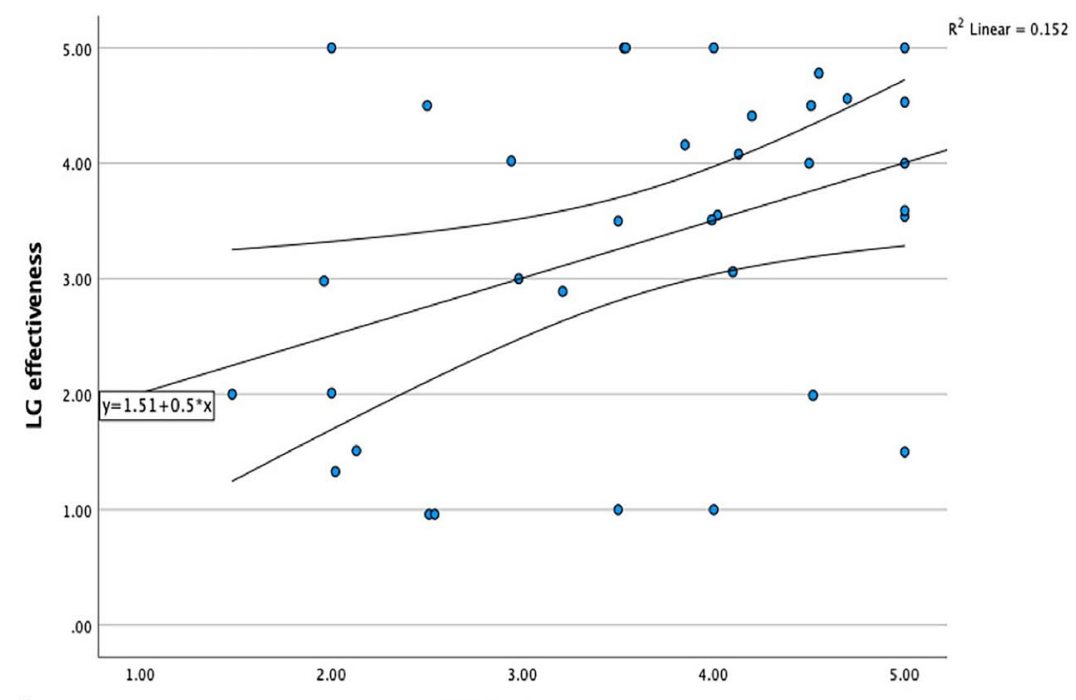

A.

OV effectiveness

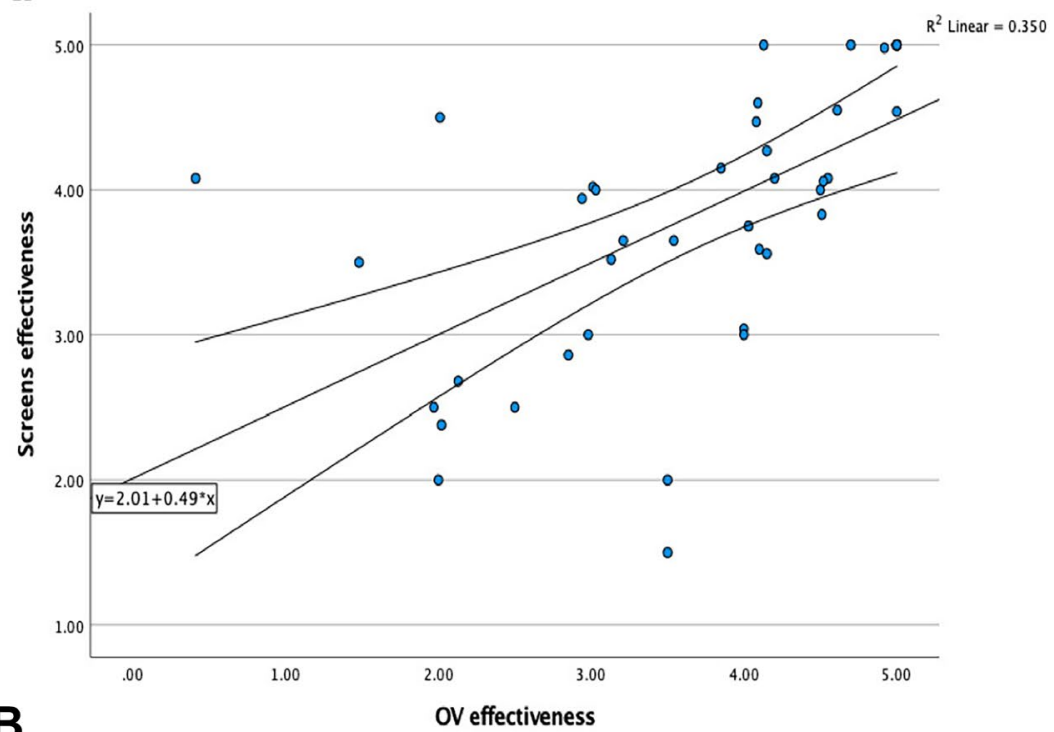

B.

OV effectiveness

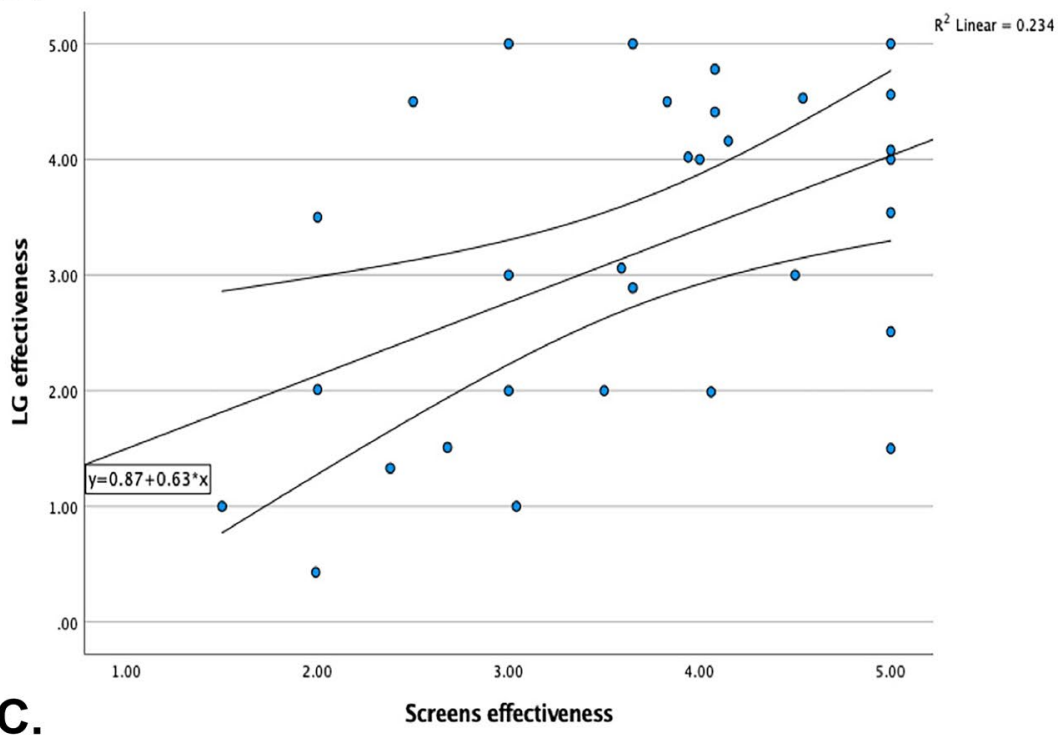


they already had to watch. One student noted that “... psychologically speaking, when they are shorter, you can go through quicker, check more things off your to-do list, then I probably would have used them more."

2) What aspects of the Complete Anatomy screens were most helpful and what was less helpful?

Our data shows that most of the students agreed that the Complete Anatomy screens served as a moderately beneficial study resource. The most helpful aspects of the Complete Anatomy screens were the ability to manipulate the screen and self-quiz on structures. One student said that: "It wasn't prelabeled so I would try to quiz myself and... identify the muscles then click the label." Like the orientation videos, the students preferred to have cadaveric images next to the Complete Anatomy screens. Students used the animated 3D version of the cadaver images as a stepping stone before studying cadaveric images. For example, one student noted: "I found myself using CA... to learn the structures and how they related to each other and then once I felt like I sort of got that I would then move to the cadaver pictures."

There were two limitations of the Complete Anatomy screens. Students noted there was an abundant number of resources and complained of resource overload. They said that while Complete Anatomy screens were helpful, there were other resources that were more beneficial and it was difficult to decide which resource best suited their learning style. The main complaint of the Complete Anatomy screens was difficulty manipulating the program itself. "I tended to not use that resource just because it felt like... I was spending too much time like fighting with the program when I could have just been learning it a different way."

3) What aspects of the lab guides were most helpful and what was less helpful?

The students' responses suggested that most did not utilize the laboratory guides for their intended purpose. The laboratory guides contain cadaveric images, noncadaveric images, and descriptions of structures to be viewed during laboratory sessions. They were intended to be used concurrently during lab in order to help students quickly identify structures without the assistance of a prosector. Overall, students found the lab guides to be an effective resource in preparing for the lecture exam and lab practical but not effective as a resource to identify structures during lab. During the beginning of the course, students noted they utilized the laboratory guides as intended. As the course progressed, students indicated they relied less on the laboratory guides and relied more on the prosectors themselves. Students did not understand the role of the prosectors and the prosectors were unsure of how independent students wanted to be. One student said: "Over time, they (prosectors) took a much more assertive role in managing that time (during lab), what was on the iPad didn't matter as much anymore."

Students found the laboratory guides more beneficial as a post-lab study resource for the lab practical exam. The vast majority of students noted that the cadaveric images present in the guides were helpful. Many students noted they used the cadaveric images from Rohen, Yokochi, and Lutjen-Drecoll [27] for self-quizzing in preparation for their exams. Students commented: "I used it as practice for the practical exam portion." "Photos with the structure list really helped... I can't say I really read more than a few words of the text." Overall, students found the lab guides to be an effective resource in preparing for the lecture exam and laboratory practical but not effective as a resource to identify structures during lab.

Critiques of the laboratory guides were directed towards their organization. A few students noted the laboratory guides were too lengthy and similar to the Complete Anatomy screens, found them to be an overload of information. Most students did not utilize the lab guides during lab was due to time constraint during laboratory sessions. They preferred the interaction with their prosectors and classmates as opposed to learning off an electronic device. "Prosector reviews were the most helpful thing, someone could actually orient me to what I was seeing and explain their techniques."

4) What aspects of the Zoom recitation sessions were most helpful and what was less helpful?

Participants across the focus groups overwhelmingly agreed Zoom recitation sessions were the one of most valuable resources available. One student said "I think they were one of the most helpful things in anatomy," and, "I would have not done well on the practical if I didn't attend those Zoom sessions." What made these Zoom recitations so successful compared to other resources?

Zoom is convenient and easily accessible. Students cited the convenience as being extremely pertinent because it saved them valuable time needed for studying. One student said, "I would be studying at my desk all day anyways so all I had to do was just click on the Zoom whereas if they were [in-person] then... I'd have to walk to campus." Another said, "I really liked that they were on zoom... for purely the convenience factor."

The Zoom recitation sessions were not only easily accessible to students, but students had the ability to request topics for the sessions. This degree of flexibility is not usually available with traditional in-person open lab sessions.

The variety of asynchronous teaching techniques used on Zoom contributed to suiting different learning styles. Zoom's various features, including screen shar- 
ing, small group break out rooms, and others, allowed the prosectors to easily cater to different learning styles. One student said, "...I definitely think that zoom is a good platform... because they can share their screen and they can send the screenshots after and you can see them (drawing during the session)."

Finally, the Zoom recitation sessions were considered so successful due to the prosectors' explanations of the anatomy. Students appreciated the guidance and ability to obtain different viewpoints from numerous prosectors, as well as their responsiveness to real-time feedback. One student said, "I also really liked that the med students accepted suggestions for how to make it better for us."

\section{Survey Discussion}

There are several factors that may contribute to correlated rankings of two different resources. Many orientation videos are created from Complete Anatomy screens. Hence, students who favor studying from the Complete Anatomy screens may feel inclined to correlate positive rankings between orientation videos and screens. Cadaveric images are present in all resources. Laboratory guides contain the greatest number of cadaveric images followed by a tie between screens and videos. Based on the number of cadaveric images, students who attribute positive rankings to laboratory guides may feel less inclined to positively rate screens or videos.

Although most students who rated one resource highly likewise rated other resources of equal value, this was shown not to always be the case. Video effectiveness and screen effectiveness were shown to be significantly correlated with one another (Spearman's rank correlation coefficient $=0.734$ ). However, there were several outliers from the regression line. One data point showed a student who rated video effectiveness at $<1.0$ but rated screen effectiveness $>4.0$ on the Likert scale. This finding was unexpected because the 3D models displayed in the orientation videos were created based on the Complete Anatomy screens. We predicted that students who rated the videos highly would also rate the Complete Anatomy screens as equally if not more effective. There could be many factors for this particular negative rating of video effectiveness, most likely related to the framework of the video itself. Videos could have been too lengthy, unnecessarily challenging, or perhaps even too simplistic for that student.

Lab guide effectiveness was also positively correlated with screen effectiveness however to a lesser degree (Spearman's rank correlation coefficient $=0.430)$. One student rated screens as very effective (5.0) but rated lab guides negatively $(<2.0)$. Both resources utilize cadaver and 3D images. One reason could be that Complete Anatomy screens provided the user with more freedom, enabling them to manipulate the $3 \mathrm{D}$ model. It is difficult to ascertain the reasoning behind this. Out of the three resources compared, the effectiveness between lab guides and orientation videos was shown to be least positively correlated (Spearman's rank correlation coefficient $=0.344$ ). There were two interesting outliers. One student rated videos highly (5.0) but found the lab guides to be ineffective $(<2.0)$. In contrast, the other student had a positive response for the lab guide (5.0) yet rated the videos negatively (2.0). The difficulty of the resources most likely played a role in these ratings. Lab guides are more in depth and conceptually more difficult than the cursory orientation videos. Prior knowledge is known to make a significant difference in performance and learning [13, 29, 32]. Therefore, we conclude that students with prior anatomical experience would be more inclined to rate videos negatively and lab guides positively.

\section{Focus Group Discussion}

In this study, the majority of students agreed that the computer-based course materials provided strongly complemented their learning of gross anatomy. Students chose to prioritize certain resources over others. Focus group responses indicated that students prioritized resources that were easily accessible. Above all, students prioritized resources that they believed would yield them the greatest benefit in succeeding in their lecture and laboratory examinations. All students strongly advocated for the Zoom recitation sessions. Students also found orientation videos and Complete Anatomy screens to complement the laboratory setting. While laboratory guides were received positively, there were mixed opinions on when (i.e., before or after) these were used.

It is important to acknowledge that all three of the resources were created by the teaching staff at BUSM. Medical students may be more likely to select resources that are recommended by peers [15]. Hence, students who prioritized certain resources may have done so purely based on peer recommendation. Financial cost has been shown to be a significant factor that students consider when selecting which resources to use [4]. However, as resources were available to students free of charge, issues of cost were not a factor in this case. Based on the responses, there are several factors that all students consider when selecting study material.

Because the lab practical exam was administered online in 2020, students were not tested on cadavers in person, as opposed to a traditional anatomy practical. The online lab practical contained both digital anatomy images from Complete Anatomy and cadaveric images from Rohen et al. [27]. Students typically find it more difficult to orient themselves to cadaveric structures as opposed to 3D structures. As such, the majority of students strongly preferred using cadaveric images as their primary resource for the lab practical. Students need to be able to identify cadaveric structures 
in order to succeed in their exams. All the resources created for the course strongly supplemented the student's ability to correctly identify structures in some way. Orientation videos and Complete Anatomy screens provided a preface to cadaveric structures. They allowed students to identify structures more quickly in the lab, which in turn made it easier to identify a 2D image in an exam. Lab guides also contained many cadaveric images. Data from a study looking at 3D animations to teach the musculoskeletal system has shown to be an effective tool in teaching anatomy [14]. However, computer-based anatomy has been shown to have limitations compared to in-person teaching [16].

Proficiency in identifying prosections during lab is not a top priority for some students because it is a smaller component of the grade compared to their lab exam. However, most students noted that learning from prosections complemented their learning of gross anatomy and prepared them for the online practical. It was surprising that the Complete Anatomy screens were not rated higher as a learning resource since the lab practical exam contained images from the Complete Anatomy screens. Again, financial concerns can be eliminated as a variable because the application was available to all students free of cost. We believe the critiques of Complete Anatomy screens were largely due to the limitations of the application itself. Students noted that the application was difficult to access and the application would often shut down during use. Other studies have shown that 3D atlases can be neglected due to functional inconvenience [24].

The Zoom sessions were by far the learning resource students most strongly touted. The high accessibility (every day of the week from early morning to late evening) and convenience (online vs in-person) of the Zoom sessions were popular among the students. Because the students also had the ability to request topics for review, they were able to allot their time to study other material while a more knowledgeable other taught them more difficult topics.

\section{Limitations}

Some limitations of the study included abiding by social distancing guidelines imposed by COVID-19 which resulted in students receiving far less time to study in the lab. The survey response rate was also lower than anticipated (50\%) as well as the number of participants recruited for the focus group.

\section{Conclusion}

We compared performance data from both written and laboratory practical examinations from course results in 2019 and 2020 in order to gauge if students adequately learned the material. We found that the students who took the anatomy course during the onset of COVID had a slightly higher overall average score in all three modules of the course than compared to the 2019 students. The overall average of the 2020 students was an $87.41 \%$ and the 2019 students scored marginally lower $-86.60 \%$. The data from this study demonstrates that although the new course materials designed to be used during the COVID-19 pandemic were able to effectively and efficiently reinforce student learning of gross anatomy, the way resources were utilized was unanticipated and unique. Orientation videos and Complete Anatomy screens were utilized to preview laboratory material while laboratory guides and Zoom recitation sessions were utilized to review laboratory material. As challenging as the pandemic has been, it has also provided anatomists with the unique opportunities to reevaluate their anatomy course, contribute to digital anatomy education, and contribute to the ongoing efforts to improve anatomy pedagogy during the pandemic.

Supplementary Information The online version contains supplementary material available at https://doi.org/10.1007/s40670-022-01524-x.

Acknowledgements The authors wish to thank the individuals who donate their bodies and tissues for the advancement of education and research and for the following funding source: Boston University School of Medicine, Department of Anatomy and Neurobiology Start-up Fund.

Author Contribution *Authors contributed equally. *DL and *BB drafted the manuscript, developed conceptual and theoretical frameworks, and collected and analyzed data. RZDDS contributed to the interpretation of results and data analysis. DC and JZ helped with data analysis. AZ is the course director and helped supervise the study. JW supervised and directed the study. All authors provided integral feedback of the manuscript.

Funding Boston University School of Medicine, Department of Anatomy and Neurobiology Start-Up Fund.

Availability of Data and Material All data and material are available upon request.

\section{Declarations}

Ethics Approval IRB Number: H-40805.

Consent to Participate All participants in this study took part voluntarily and were informed their responses would remain anonymous.

Consent for Publication All participants in this study took part voluntarily and were informed their responses would remain anonymous.

Conflict of Interest The authors declare no competing interests.

\section{References}

1. Ahmed K, Rowland S, Patel V, Khan RS, Ashrafian H, Davies $\mathrm{DC}$, et al. Is the structure of anatomy curriculum adequate for safe medical practice? Surgeon. 2010;8(6):318-24. 
2. Azer SA, Eizenberg N. Do we need dissection in an integrated problem-based learning medical course? Perceptions of first- and second-year students. Surg Radiol Anat. 2007;29(2):173-80.

3. Cho MJ, Hwang YI. Students' perception of anatomy education at a Korean medical college with respect to time and contents. Anat Cell Biol. 2013;46(2):157-62.

4. Choi-Lundberg DL, Low TF, Patman P, Turner P, Sinha SN. Medical student preferences for self-directed study resources in gross anatomy. Anat Sci Educ. 2016;9(2):150-60.

5. Crow SM, O'Donoghue D, Vannatta JB, Thompson BM. Meeting the family: promoting humanism in gross anatomy. Teach Learn Med. 2012;24(1):49-54.

6. Drake RL, McBride JM, Lachman N, Pawlina W. Medical education in the anatomical sciences: the winds of change continue to blow. Anat Sci Educ. 2009;2(6):253-9.

7. Drake RL, McBride JM, Pawlina W. An update on the status of anatomical sciences education in United States medical schools. Anat Sci Educ. 2014;7(4):321-5.

8. Estai M, Bunt $\mathrm{S}$. Best teaching practices in anatomy education: a critical review. Ann Anat. 2016;208:151-7.

9. Finegan O, Fonseca S, Guyomarc'h P, Mendez MDM, Gonzalez JR, Tidball-Binz M, et al. International Committee of the Red Cross (ICRC): General guidance for the management of the dead related to COVID-19. Forensic Science International: Synergy. 2020;2:129-37.

10. Franchi T. The impact of the Covid-19 pandemic on current anatomy education and future careers: a student's perspective. Anat Sci Educ. 2020;13(3):312-5.

11. Ghosh SK, Kumar A. Building professionalism in human dissection room as a component of hidden curriculum delivery: a systematic review of good practices. Anat Sci Educ. 2019;12(2):210-21.

12. Goldie JG. Connectivism: a knowledge learning theory for the digital age? Med Teach. 2016;38(10):1064-9.

13. Hailikari T, Katajavuori N, Lindblom-Ylanne S. The relevance of prior knowledge in learning and instructional design. Am J Pharm Educ. 2008;72(5):113.

14. Hoyek N, Collet C, Di Rienzo F, De Almeida M, Guillot A. Effectiveness of three-dimensional digital animation in teaching human anatomy in an authentic classroom context. Anat Sci Educ. 2014;7(6):430-7.

15. Judd T, Elliott K. Selection and use of online learning resources by first-year medical students: cross-sectional study. JMIR Med Educ. 2017;3(2):e17.

16. Khot Z, Quinlan K, Norman GR, Wainman B. The relative effectiveness of computer-based and traditional resources for education in anatomy. Anat Sci Educ. 2013;6(4):211-5.

17. Kramer B, Billings B, Moxham B, Winkelmann A. IFAA best practice guidelines for body donation programmes during the novel coronavirus pandemic. Johannesburg, South Africa: International Federation of Associations of ... 2020.
18. McLachlan JC. New path for teaching anatomy: living anatomy and medical imaging vs. dissection. Anat Rec B New Anat. 2004;281(1):4-5.

19. Nnodim JO. Learning human anatomy: by dissection or from prosections? Med Educ. 1990;24(4):389-95.

20. Noble H, Mitchell G. What is grounded theory? Evid Based Nurs. 2016;19(2):34-5.

21. Older J. Anatomy: a must for teaching the next generation. Surgeon. 2004;2(2):79-90.

22. Onigbinde O. COVID-19 pandemic era: how risky is the continuous usage of cadavers for teaching and research? Elsevier. 2021.

23. Organization WH. Infection prevention and control for the safe management of a dead body in the context of COVID-19: interim guidance, 4 September 2020. World Health Organization. 2020.

24. Park S, Kim Y, Park S, Shin JA. The impacts of three-dimensional anatomical atlas on learning anatomy. Anat Cell Biol. 2019;52(1):76-81.

25. Patel KM, Moxham BJ. Attitudes of professional anatomists to curricular change. Clin Anat. 2006;19(2):132-41.

26. Ravi KS. Dead body management in times of Covid-19 and its potential impact on the availability of cadavers for medical education in India. Anat Sci Educ. 2020;13(3):316-7.

27. Rohen JW, Yokochi C, Lütjen-Drecoll E. Color atlas of anatomy: a photographic study of the human body: Schattauer Verlag. 2006.

28. Shidham VB, Frisch NK, Layfield LJ. Severe acute respiratory syndrome coronavirus 2 (the cause of COVID 19) in different types of clinical specimens and implications for cytopathology specimen: an editorial review with recommendations. Cytojournal. 2020;17.

29. Tedman RA, Alexander H, Massa H, Moses D. Student perception of a new integrated anatomy practical program: does students' prior learning make a difference? Clin Anat. 2011;24(5):664-70.

30. Topp KS. Prosection vs. dissection, the debate continues: rebuttal to Granger. Anat Rec B New Anat. 2004;281(1):12-4.

31. Turney BW. Anatomy in a modern medical curriculum. Ann R Coll Surg Engl. 2007;89(2):104-7.

32. Wetzels SA, Kester L, van Merrienboer JJ, Broers NJ. The influence of prior knowledge on the retrieval-directed function of note taking in prior knowledge activation. Br J Educ Psychol. 2011;81(Pt 2):274-91.

33. Winichakoon P, Chaiwarith R, Liwsrisakun C, Salee P, Goonna A, Limsukon A, et al. Negative nasopharyngeal and oropharyngeal swabs do not rule out COVID-19. J Clin Microbiol. 2020;58(5).

34. Wisco JJ, Young S, Rabedeaux P, Lerner SD, Wimmers PF, Byus $\mathrm{C}$, et al. Student perceived value of anatomy pedagogy, part I: prosection or dissection? J Med Educ Curric De. 2015;2:15-20.

Publisher's Note Springer Nature remains neutral with regard to jurisdictional claims in published maps and institutional affiliations. 